ESAIM: PROCEEDINGS, July 2007, Vol.18, 163-180

Jean-Frédéric Gerbeau \& Stéphane Labbé, Editors

\title{
QUELQUES MÉTHODES DE PARAMÈTRE D'ORDRE AVEC APPLICATIONS À LA MODÉLISATION DE PROCESSUS CANCÉREUX
}

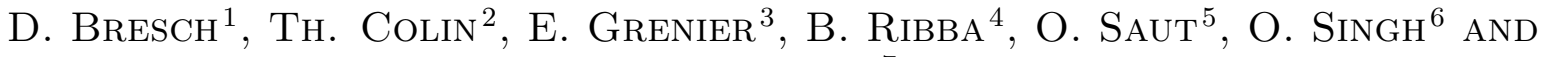 \\ C. VERDIER ${ }^{7}$
}

\begin{abstract}
This paper deals with level set, phase field, diffusive interface methods or more generally methods with scalar order parameter. The goal of these lines is to provide to the reader a unified overview of such technics with various recent applications of such methods to cancerology. We also present some actual open questions.

Résumé. Nous présentons succintement dans ce papier quelques méthodes de paramètre d'ordre scalaire. Le but est de donner au lecteur une vue rapide mais unifiée de ces techniques sans trop de détails avec diverses applications de ces méthodes à la modélisation des processus cancéreux. Nous présentons également quelques questions ouvertes intéressantes.
\end{abstract}

\section{INTRODUCTION}

Nous présentons succintement dans ce papier les méthodes de lignes de niveaux, champ de phase, interface diffuse ou plus généralement quelques méthodes avec paramètre d'ordre scalaire. Le but est de donner au lecteur une vue unifiée et rapide de ces techniques avec diverses applications à la cancérologie. Nous présentons quelques questions de modélisation intéressantes (choix de viscosité, analogie de modèles, etc...). On parlera par exemple de modèle de Cahn-Hilliard, Allen-Cahn, Korteweg pour les problèmes multifluides ou multiphases. On renvoie le lecteur à [40], et les références citées à l'intérieur, pour un exposé complémentaire sur les méthodes numériques pour interfaces libres ainsi qu'à [29] pour un exposé sur les modèles d'interface diffuse en mécanique des fluides.

Le plan de notre exposé sera le suivant : dans une première partie, nous exposons la méthode de lignes de niveaux pour deux fluides non miscibles alors que la deuxième partie concerne la modélisation de fluides

\footnotetext{
${ }^{1}$ LAMA, UMR 5127 CNRS, université de Savoie 73376 Le Bourget du lac cedex, France. Email: didier.bresch@univ-savoie.fr

2 Mathématiques Appliquées de Bordeaux, CNRS UMR 5466 and INRIA futurs, Université de Bordeaux I, 251 avenue de la libération, 33405 Talence cedex, France. Email: thierry.colin@math.u-bordeaux.fr

3 U.M.P.A., École Normale Supérieure de Lyon, 46, allée d'Italie, 69364 Lyon Cedex 07, France. Email: egrenier@umpa.enslyon.fr

${ }^{4}$ Université de Lyon, Lyon, F-69003, France; Université Lyon 1, Ciblage Thérapeutique en Oncologie (EA3738), Faculté de Médecine Lyon-Sud, Oullins, F-69921, France

5 Mathématiques Appliquées de Bordeaux, CNRS UMR 5466 and INRIA futurs, Université de Bordeaux I, 251 avenue de la libération, 33405 Talence cedex, France. Email : olivier.saut@math.u-bordeaux.fr

${ }^{6}$ New Jersey Institute of Technology (NJIT), Department of Mechanical Engineering, University Heighs, New Jersey 07102, USA. Email: puspendra.singh@njit.edu

${ }^{7}$ Laboratoire de Spectrométrie Physique (SPECTRO), Université Joseph-Fourier UMR 5588, 140 avenue de la physique, 38402 Saint Martin d'Hères cedex, France. Email : claude.verdier@ujf-grenoble.fr
}

(C) EDP Sciences, SMAI 2007 
miscibles. Dans une troisième partie, nous essaierons de sensibiliser le lecteur à la problèmatique du choix de l'énergie libre dans la recherche de forme d'équilibre et dans la dynamique de ces formes. Nous introduisons pour cela les modèles à interface diffuse de type Korteweg. La cinquième partie concerne des exemples d'applications à la modélisation de processus cancéreux de toutes les méthodes décrites dans les parties précédentes.

\section{Méthode De lignes de niveaux pour DeUX FLuides NON-Miscibles.}

Nous présentons dans cette section un exemple d'utilisation de la méthode de lignes de niveaux pour rechercher une interface entre deux fluides non miscibles. L'exposé qui suit est classique mais décrira les principales étapes, voir par exemple [43]. Il est important de préciser que cette technique peut être appliquée à d'autres équations aux dérivées partielles : ce sera le cas dans l'une des applications en modélisation du processus d'invasion en cancérologie, que l'on donnera par la suite, où l'on considèrera des équations aux dérivées partielles pour milieux poreux avec une méthode de lignes de niveaux pour le suivi de la déformation et de la dégradation de la membrane extra-cellulaire. La différence principale proviendra de l'énergie élastique d'interface à considérer avec un champ de vecteur qui ne sera pas incompressible.

Considérons l'écoulement de deux fluides non-miscibles dans un domaine $\Omega \subset \mathbb{R}^{3}$. $\grave{\mathrm{A}}$ un instant $t$, notons $\Omega_{1}(t) \subset \Omega$ le sous-domaine contenant le premier fluide et $\Omega_{2}(t)$ contenant le second, si bien que $\bar{\Omega}=\overline{\Omega_{1}(t)} \cup \overline{\Omega_{2}(t)}$. L'interface entre les deux fluides sera noté $\Gamma(t)=\partial \Omega_{1}(t) \cap \partial \Omega_{2}(t)$.

$\grave{\mathrm{A}} t=0$, on suppose que l'interface est une surface paramétrée par $\chi_{0}$ connue

$$
\Gamma(0)=\left\{x=\chi_{0}(\xi) \in \Omega ; \xi \in[0,1]^{2}\right\}
$$

À $t>0$, l'interface sera décrite par une surface paramétrée par $\chi$ inconnue:

$$
\Gamma(t)=\left\{x=\chi(\xi, t) \in \Omega ; \xi \in[0,1]^{2}\right\} .
$$

Sachant que la position $\chi(\xi, t)$ de l'interface est convectée par la vitesse $u$ du fluide, nous en déduisons que

$$
\frac{d \chi}{d t}(\xi, t)=u(\chi(\xi, t), t), \quad \chi(\xi, t=0)=\chi_{0}(\xi)
$$

Sur l'interface, les équations sont

$$
[u]=0, \quad\left[\sigma_{\text {tot }} n\right]=\alpha \kappa n
$$

avec $\kappa=\operatorname{div} n$ et où $[\phi]=\left.\phi\right|_{\Omega_{2}(t)}-\left.\phi\right|_{\Omega_{1}(t)}$ est le saut d'une quantité $\phi$ à travers l'interface $\Gamma(t), \sigma_{\text {tot }}$ représente le tenseur des contraintes totales, $n$ est la normale unitaire sur $\Gamma(t)$ orientée de $\Omega_{1}(t)$ vers $\Omega_{2}(t)$, $\alpha$ est le coefficient de tension de surface et $\kappa(x, t)$ est la courbure de la surface $\Gamma(t)$. Ces relations expriment que le champ de vitesses et les contraintes tangentielles sont continus à travers l'interface tandis que les contraintes normales sont discontinues.

Nous supposons que la densité du premier (resp. deuxième) fluide, notée $\rho_{1}$ (resp. $\rho_{2}$ ), est constante, si bien que la conservation de la masse conduit à

$$
\left.\operatorname{div} u=0 \text { dans } \Omega_{i}(t) \times\right] 0, T[, i=1,2
$$

ce qui se réduit à

$$
\operatorname{div} u=0 \text { dans } \Omega \times] 0, T[.
$$

La conservation de la quantité de mouvement pour le $i$ ème fluide s'écrit

$$
\left.\rho_{i} \frac{d u_{i}}{d t}-\operatorname{div} \sigma_{\text {tot }}=\rho_{i} g \text { dans } \Omega_{i} \times\right] 0, T[, i=1,2 .
$$


De plus nous supposons les deux fluides newtoniens. Le tenseur des contraintes totales est alors donné par

$$
\sigma_{\mathrm{tot}}=-p \operatorname{Id}+2 \eta D(u)
$$

avec $D(u)=\left(\nabla u+{ }^{t} \nabla u\right) / 2$ désigne le tenseur de déformation et

$$
\eta=\eta_{1} \text { dans } \Omega_{1}(t), \quad \eta=\eta_{2} \text { dans } \Omega_{2}(t)
$$

avec $\eta_{1}$ et $\eta_{2}$ deux constantes positives.

Le problème est complété par des conditions initiales et aux limites et revient à trouver $\chi, u$ et $p$ solutions des équations données précédemment avec les conditions aux bords et initiales adéquates.

La méthode de lignes de niveaux consiste à représenter l'interface $\Gamma(t)$ par la courbe de niveau zéro d'un paramètre d'ordre $\phi$ défini sur tout le domaine. En convectant le paramètre d'ordre, l'interface se retrouve elle-même convectée à la bonne vitesse. Pour la mécanique des fluides, ces méthodes sont appliquées et des outils de plus en plus pointus sont développés pour envisager des écoulements complexes. Considérons donc $\phi$ une fonction définie dans $\Omega \times] 0, T[$ telle que

$$
\phi(t, x)=0 \text { si } x \in \Gamma(t), \quad \phi(t, x)<0 \text { si } x \in \Omega_{1}(t), \quad \phi(t, x)>0 \text { si } x \in \Omega_{2}(t) .
$$

Remarquons que $\Gamma(t)$ est alors caractérisé par

$$
\Gamma(t)=\{x \in \Omega: \phi(t, x)=0\} .
$$

En posant $\rho=\rho_{1}$ dans $\Omega_{1}(t)$ et $\rho=\rho_{2}$ dans $\Omega_{2}(t)$ alors les équations de quantité de mouvement peuvent s'écrire de manière condensée

$$
\rho \frac{d u}{d t}-\operatorname{div} \sigma_{\text {tot }}+\alpha \kappa n \delta(\phi)=\rho g
$$

où $\delta$ désigne la mesure de Dirac portée par l'interface séparant les deux fluides et $\kappa$ le coefficient de tension de surface à l'interface. On considèrera par la suite $\widetilde{\kappa}$ une extension de $\kappa$ sur tout le domaine.

On sait que l'on a l'équation suivante sur $\phi$

$$
\left.\partial_{t} \phi+u \cdot \nabla \phi=0 \text { sur } \Gamma(t) \times\right] 0, T[.
$$

L'extension de $\phi$ de $\Gamma(t)$ à tout $\Omega$ étant arbitraire, nous prolongeons cette équation à tout $\Omega$

$$
\left.\partial_{t} \phi+u \cdot \nabla \phi=0 \operatorname{sur} \Omega \times\right] 0, T[
$$

Le problème revient donc à trouver $\phi, u$ et $p$ tels que $\phi$ satisfait l'équation de transport sur tout $\Omega$ et $u$ et $p$ satisfont l'équation de quantité de mouvement

$$
\left.\rho(\phi)\left(\partial_{t} u+u \cdot \nabla u\right)-2 \operatorname{div}(\eta(\phi) D(u))+\nabla p+\alpha \widetilde{\kappa} \delta(\phi) \frac{\nabla \phi}{|\nabla \phi|}=\rho(\phi) g \text { dans } \Omega \times\right] 0, T[
$$

où $\rho(\phi)=\rho_{1}+\left(\rho_{2}-\rho_{1}\right) H(\phi), \eta(\phi)=\eta_{1}+\left(\eta_{2}-\eta_{1}\right) H(\phi)$. La fonction d'Heaviside est définie pour presque tout $\phi \in \mathbb{R}$ par

$$
H(\phi)=1 \text { si } \phi>0, \quad H(\phi)=0 \text { sinon. }
$$

Une méthode couramment utilisée en pratique consiste à utiliser une propriété d'étalement d'interface. Plus précisemment on utilise la propriété suivante : si $\xi$ est une fonction à support compact, de masse un, alors quand $\epsilon$ tends vers 0 , la fonction $|\nabla \phi| \frac{1}{\epsilon} \xi\left(\frac{\phi}{\epsilon}\right) \rightarrow \delta_{\{\phi=0\}}$. Ainsi $\epsilon>0$ devient un nouveau paramètre d'approximation du problème. 
Afin de définir une fonction adéquate, on remplace la fonction de Heaviside $H$ par une fonction $H_{\epsilon}$ régulière. On peut par exemple considérer $H_{\epsilon}=0$ si $\phi<-\epsilon, H_{\epsilon}=1$ si $\phi>\epsilon$ et $H_{\epsilon}=(1+\phi / \epsilon+\sin (\pi \phi / \epsilon) / \pi) / 2$ si $|\phi| \leq \epsilon$. Ce qui conduit à définir

$$
\delta_{\epsilon}(\phi)=(1+\cos (\pi \phi / \epsilon)) / 2 \epsilon \text { si }|\phi| \leq \epsilon, \quad \text { ou } \delta_{\epsilon}(\phi)=0 \text { sinon. }
$$

Beaucoup d'autres choix pour $\delta_{\epsilon}$ peuvent être faits et ont par exemple été considérés dans [53].

On remplace $\rho$ et $\eta$ par

$$
\begin{aligned}
& \rho_{\epsilon}=\rho_{1}+\left(\rho_{2}-\rho_{1}\right) H_{\epsilon}(\phi) \\
& \eta_{\epsilon}=\eta_{1}+\left(\eta_{2}-\eta_{1}\right) H_{\epsilon}(\phi) .
\end{aligned}
$$

L'épaisseur de l'interface est alors $2 \epsilon$ et s'interprète comme une zone de mélange des deux fluides avec $1-H_{\epsilon}(\phi)$ de fluide 1 et $H_{\epsilon}(\phi)$ de fluide 2 . L'interface raide de séparation des fluides a donc été remplacée par une interface diffuse.

La fonction de niveau $\phi$ est initialisée comme une fonction distance signée. En particulier nous avons $|\nabla \phi|=1$ à l'instant initial. Cependant cette propriété n'est pas conservée au cours du temps. Il se peut que $|\nabla \phi|$ devienne très grand, par exemple quand une goutte vient toucher un plan. Le problème s'énonce alors ainsi : À un instant fixé, connaissant $\phi(t)$, construire une distance signée qui ait la même isovaleur zéro : $\Gamma(t)=\{\widetilde{\phi}(t)=0\}$. Alors $\widetilde{\phi}(t)$ est la solution stationnaire $\psi_{t}(\tau=+\infty)$ de

$$
\partial_{\tau} \psi_{t}(x, \tau)=\operatorname{signe}(\phi(t, x))\left(1-\left|\nabla \psi_{t}(\tau, x)\right|\right)
$$

où $\tau$ est un pseudo-temps et $\operatorname{signe}(\phi)=-1$ si $\phi<0,0$ si $\phi=0$ et 1 si $\phi>0$. Puisque signe $(0)=0$, pour tout $\tau \geq 0, \psi_{t}(\tau, x)$ possède la même isovaleur zéro que $\phi(t)$. Ainsi il reste à résoudre le problème précédent jusqu'à obtenir un état stationnaire. Notons qu'il suffit d'obtenir une fonction distance signée, c'est-à-dire de gradient unité uniquement dans un voisinage du front.

Les techniques numériques pour discrétiser avec précision les équations décrites précédemment ont fait l'objet de multiples études : citons par exemple les schémas ENO, WENO, WENO5, cf. [43], [30], [44], [50]. Pour la microfluidique, les interfaces sont telles que certains outils sophistiqués ne sont pas nécessaires, un schéma WENO5 pour un paramètre d'ordre régulier (distance signée à l'interface) donne de très bon résultats. La microfluidique soulève des difficultés numériques d'une tout autre nature que pour les écoulements classiques. Il s'agit d'écoulements numériques parasites qui apparaissent à l'interface liés au terme de tension de surface. Par le choix de pas de temps suffisamment petits, on fait disparaître ces écoulements parasites, en revanche le coût de calcul devient déraisonnable. Une méthode, récemment mise au point dans [26], spécifique à la microfluidique élimine l'instabilité pour un coût de calcul raisonnable, elle est généralisable à d'autres méthodes de suivi d'interface. Cette méthode se base sur un splitting des écoulements et la condition de stabilité restrictive sur le pas de temps n'est appliquée qu'à une partie de l'écoulement. Cette méthode est importante lors de la simulation de mouvements de cellules à l'intérieur des vaisseaux sanguins pour comprendre les phénomènes d'intravasation et extravasation. On renvoie également à [4], [7] et [8] pour des applications de champ de phase à des problèmes de déplacements de vésicules.

\section{Modèles de MÉLANGe POUR LES Fluides.}

Pour les fluides miscibles, on comprend bien que l'on ne va pouvoir avoir recours aux méthodes de lignes de niveaux. Nous devons donc choisir un tout autre paramètre d'ordre. Il s'agit par exemple de la fraction volumique. Les modèles d'Allen-Cahn, de Cahn-Hilliard sont des modèles classiques de ce genre (voir par exemple [9], [10], [18], [36]). On renvoie également le lecteur au livre de K.R. RAJAGOPAL et L. TAO dans [51]. On s'inspirera d'ailleurs fortement de ces travaux pour la rédaction de cette partie. Nous verrons que la mise en place d'un modèle exploitable demande une quantité importante d'hypothèses formelles. Il serait très intéressant d'essayer de justifier mathématiquement quelques modèles simples de mélange dans des configurations idéalisées. 
Dans un domaine $\Omega$, on suppose avoir deux constituants avec $\mathcal{V}_{1}$ et $\mathcal{V}_{2}$ les volumes occupés par ces deux entités dans un élément de volume $\mathcal{V}$, c'est-à-dire

$$
\mathcal{V}=\mathcal{V}_{1} \cup \mathcal{V}_{2}
$$

La fraction volumique du fluide 1 dans le volume $\mathcal{V}$ à l'instant $t$ est définie par

$$
\psi(\mathcal{V}, t)=\frac{\operatorname{mes} \mathcal{V}_{1}}{\operatorname{mes} \mathcal{V}}
$$

Cette définition étant possible pour tout voisinage $\mathcal{V}$ contenant $x \in \Omega$, on définit alors la fraction volumique en $x$ par

$$
\psi(x, t)=\lim _{\operatorname{mes} \mathcal{V} \rightarrow 0, x \in \mathcal{V}} \frac{\operatorname{mes} \mathcal{V}_{1}}{\operatorname{mes} \mathcal{V}} .
$$

La fraction volumique est à valeurs dans l'intervalle $[0,1]$. Pour des raisons de commodité, cette fraction volumique est souvent normalisée dans $[-1,1]$ :

$$
\phi=2 \psi-1
$$

Ainsi $\phi(x, t)=1$ si seul le constituant 1 est présent, et $\phi(x, t)=-1$ si seul le constituant 2 est présent en $x$ à l'instant $t$.

Soient $\rho_{1}$ et $\rho_{2}$ les densités, supposées constantes des constituants 1 et 2 , respectivement. La densité du mélange est

$$
\begin{array}{r}
\rho(x, t)=\lim _{\operatorname{mes} \mathcal{V} \rightarrow 0, x \in \mathcal{V}} \frac{\rho_{1} \operatorname{mes}\left(\mathcal{V}_{1}\right)+\rho_{2} \operatorname{mes}\left(\mathcal{V}_{2}\right)}{\operatorname{mes}(\mathcal{V})} \\
=\rho_{1} \psi+\rho_{2}(1-\psi)=\rho_{1} \frac{1+\phi}{2}+\rho_{2} \frac{1-\phi}{2}
\end{array}
$$

On supposera également que chaque constituant possède un champ de vitesse propre $u_{1}, u_{2}$. La vitesse du mélange est définie par la moyenne volumique des champs de vitesse de chaque constituant :

$$
u=\frac{1+\phi}{2} u_{1}+\frac{1-\phi}{2} u_{2} .
$$

La vitesse relative du fluide 1 par rapport au fluide 2 est notée :

$$
w=u_{1}-u_{2}
$$

Avec ces deux dernières relations, on peut exprimer les vitesses de chaque constituant en fonction de la vitesse du mélange et de la vitesse relative.

$$
u_{1}=u+\frac{1-\phi}{2} w, \quad u_{2}=u-\frac{1+\phi}{2} w .
$$

Le but est à présent de partir des équations de la mécanique des fluides sur chaque constituant pour en déduire un système d'équations aux dérivées partielles sur $u, \phi$ où $\phi$ sera le paramètre d'ordre de notre problème. Il faudra donc faire certaines hypothèses permettant de fermer le système en exprimant $w$ en fonction de $\phi$.

En réécrivant les équations de la masse sur chaque constituant en fonction de $\phi, \rho_{1}, \rho_{2}, u_{1}$ et $u_{2}$ c'est-à-dire

$$
\begin{aligned}
& \partial_{t}\left(\rho_{1} \frac{1+\phi}{2}\right)+\operatorname{div}\left(\rho_{1} \frac{1+\phi}{2} u_{1}\right)=0 \\
& \partial_{t}\left(\rho_{2} \frac{1-\phi}{2}\right)+\operatorname{div}\left(\rho_{2} \frac{1-\phi}{2} u_{2}\right)=0
\end{aligned}
$$


et en rappelant que $\rho_{1}$ et $\rho_{2}$ sont supposés constants, on trouve les deux équations suivantes

$$
\operatorname{div} u=0
$$

et

$$
\partial_{t} \phi+u \cdot \nabla \phi+\operatorname{div}\left(\frac{1-\phi^{2}}{2} w\right)=0 .
$$

En ce qui concerne les équations de quantité de mouvement, les choses sont plus complexes. On supposera les forces extérieures de la forme

$$
f_{1}=\rho_{1} \frac{1+\phi}{2}\left(g-\nabla \mu_{1}\right)-\xi(\phi) w, \quad f_{2}=\rho_{2} \frac{1-\phi}{2}\left(g-\nabla \mu_{2}\right)+\xi(\phi) w
$$

où $\xi(\phi)$ est le coefficient de frottement d'un constitutant sur l'autre et $\mu_{1}$ et $\mu_{2}$ les potentiels thermodynamiques des deux phases donnés par

$$
\mu_{1}=\frac{1-\phi}{2} \mu, \quad \mu_{2}=\frac{1+\phi}{2} \mu .
$$

Les derniers termes représentent des termes de frottement et les termes $\mu_{1}$ et $\mu_{2}$ sont les potentiels thermodynamiques d'échange entre espèces. Donner la forme de ces entités et donc a fortiori de $\mu$ conditionnera le modèle considéré : Cahn-Hilliard, Allen-Cahn etc...

On supposera de plus que les pressions partielles $p_{1}$ et $p_{2}$ de chaque phase sont reliées à la pression totale du mélange $p=p_{1}+p_{2}$ via

On supposera également que

$$
p_{1}=\frac{1+\phi}{2} p, \quad p_{2}=\frac{1-\phi}{2} p
$$

$$
\eta_{1}(\phi)=\frac{1+\phi}{2} \eta(\phi), \quad \eta_{2}(\phi)=\frac{1-\phi}{2} \eta(\phi)
$$

où $\eta(\phi)$ est la viscosité du mélange uniforme de composition $\phi$. Le potentiel thermodynamique $\mu(\phi)$ est de la forme

$$
\mu(\phi)=\mathcal{J}^{\prime}(\phi)
$$

Il dérive donc de la fonctionnelle $\mathcal{J}$ appelée énergie libre (voir section suivante). Nous citerons à titre d'exemple les fonctionnelles de Cahn-Hilliard, d'Allen-Cahn ou de Mundford Shah. Dans le cas de Cahn-Hilliard

$$
\mathcal{J}(\phi)=\int_{\Omega}\left(\frac{\beta}{2}|\nabla \phi|^{2}+\gamma j(\phi)\right) d x
$$

où $\beta, \gamma>0$ sont des constantes et $j$ est une fonction de $\mathbb{R}$ dans $\mathbb{R}$ de sorte que

$$
\mu(\phi)=\mathcal{J}^{\prime}(\phi)=-\beta \Delta \phi+\gamma j^{\prime}(\phi)
$$

La fonction $j$ est appelée potentiel de Cahn Hilliard. Un choix classique est

$$
j(\phi)=\theta_{c}\left(1-\phi^{2}\right)+\theta\{(1+\phi) \log (1+\phi)+(1-\phi) \log (1-\phi)\}, \quad-1 \leq \phi \leq 1
$$

où $\theta$ est la température du mélange, supposée constante, et $\theta_{c}$ une température critique. En particulier

$$
j^{\prime \prime}(\phi)=2 \theta_{c} \frac{\phi^{2}-\left(\theta_{c}-\theta\right) / \theta_{c}}{(1+\phi)(1-\phi)} .
$$

Ainsi $j$ est convexe dans $[-1,1]$ dès que $\theta \geq \theta_{c}$. Le cas intéressant $\theta<\theta_{c}$ prévoit que les constituants vont tendre à se séparer dans les régions où $|\phi| \leq\left(\theta_{c}-\theta\right) / \theta_{c}$, de sorte que $|\phi|>\left(\theta_{c}-\theta\right) / \theta_{c}$ presque partout dans $\Omega$. 
Une approximation souvent utilisée de ce potentiel est donnée par un développement limité de $j$ à l'ordre 4 au voisinage de $\phi=0$

$$
\widetilde{j}=\theta_{c}-\left(\theta_{c}-\theta\right) \phi^{2}+\theta \phi^{4} .
$$

On réécrit le système en $(\phi, u, w, p)$ et nous négligeons les termes en $w$ supposés petits devant les termes en $u$

$$
\begin{gathered}
\rho_{1} \frac{1+\phi}{2} u_{1}+\rho_{2} \frac{1-\phi}{2} u_{2} \approx \rho(\phi) u \\
\rho_{1} \frac{1+\phi}{2} u_{1} \otimes u_{1}+\rho_{2} \frac{1-\phi}{2} u_{2} \otimes u_{2} \approx \rho(\phi) u \otimes u \\
\sigma_{1, \text { tot }}+\sigma_{2, \text { tot }} \approx-p \mathrm{Id}+2 \eta(\phi) D(u) .
\end{gathered}
$$

On obtient alors l'équation en vitesse moyenne

$$
\begin{aligned}
\rho(\phi)\left(\partial_{t} u\right. & +u \cdot \nabla u)-\operatorname{div}(2 \eta(\phi) D(u))+\nabla p \\
& =\rho(\phi) g+\frac{\mu(\phi)}{2} \nabla \phi-\left(\rho_{1}-\rho_{2}\right) \frac{1-\phi^{2}}{4} \nabla\left(\frac{\mu(\phi)}{\rho(\phi)}\right) .
\end{aligned}
$$

On obtient également

$$
\rho_{2} \frac{1-\phi}{2} f_{1}-\rho_{1} \frac{1+\phi}{2} f_{2} \approx 0
$$

En utilisant les expressions de $f_{1}$ et $f_{2}$ cela donne

$$
w \approx-\rho_{1} \rho_{2} \frac{\left(1-\phi^{2}\right)^{2}}{4 \xi(\rho) \rho} \nabla\left(\frac{\mu}{\rho}\right) .
$$

Notons que les potentiels thermodynamiques nous ont permis de trouver une expression de la vitesse relative $w$ en fonction du paramètre d'ordre $\phi$. Il est alors possible d'obtenir le modèle en les variables $(\phi, u, p)$ suivant

$$
\begin{aligned}
& \rho(\phi)\left(\partial_{t} u+u \cdot \nabla u\right)-\operatorname{div}(2 \eta(\phi) D(u))+\nabla p \\
& =\rho(\phi) g+\frac{\mu(\phi)}{2} \nabla \phi-\left(\rho_{1}-\rho_{2}\right) \frac{1-\phi^{2}}{4} \nabla\left(\frac{\mu(\phi)}{\rho(\phi)}\right), \\
& \partial_{t} \phi+u \cdot \nabla \phi+\operatorname{div}\left(\frac{1-\phi^{2}}{2} w\right)=0, \\
& \operatorname{div} u=0, \quad w=-\rho_{1} \rho_{2} \frac{\left(1-\phi^{2}\right)^{2}}{4 \xi(\rho) \rho} \nabla\left(\frac{\mu(\phi)}{\rho}\right), \\
& \mu(\phi)=-\beta \Delta \phi+\gamma j^{\prime}(\phi),
\end{aligned}
$$

Remarque. On peut choisir une multitude d'énergie libre pour remplacer (2.4) comme par exemple celle d'AllenCahn en mécanique des fluides, celle de Mundford-Shah en imagerie, celle d'Hele shaw dans des problèmes solide-liquide.

Remarque. Un modèle de fluides miscibles est parfois utilisé pour approcher un problème de fluides immiscibles. C'est d'ailleurs ce que l'on fait lorsque l'on considère un modèle d'interface diffuse avec les modèles de Korteweg compressible ou incompressible.

Remarque. Une approche plus "simpliste" conduit à prendre $\left(1-\phi^{2}\right) w \approx D \nabla \phi$, on trouve alors une équation de réaction-diffusion sur $\phi$.

Remarque. Le système d'équations (2.6)-(2.6) a été étudié mathématiquement dans [9], [10] dans le cas de deux densités $\rho_{1}$ et $\rho_{2}$ suffisamment proches. Nous rappelons que ce système de Cahn-Hilliard est obtenu par moyennisation volumique permettant de se ramener à une condition d'incompressibilité sur la vitesse moyenne. Si l'on utilise la moyenne massique, on obtient un modèle différent avec une contrainte sur la vitesse moyenne 
du type divu $u=\Delta F(\rho)$ avec $F$ une fonction donnée liée à la loi de Fick. Le lecteur interessé pourra consulter [32] pour des applications de ces systèmes aux avalanches.

Remarque. Dans d'autres phénomènes l'existence d'un potentiel thermodynamique approprié n'est pas évident et parfois les équations de champ de phase sont construites en espérant qu'elles reproduiront le bon problème sur la limite d'interface raide. Définir une forme explicite de densité d'énergie à minimiser, par une analyse à l'échelle microscopique, et à insérer dans l'expression de l'énergie propre du matériau est standard mais n'est pas toujours possible, voir par exemple [35].

\section{Sur L'EFFET DU CHOIX DE L'ÉNERGIE LIBRE}

Afin de comprendre les phénomènes de minimisation d'énergie dans une dynamique fluide, il faut tout d'abord identifier le paramètre d'ordre adéquat à considérer. On s'intéresse ensuite au modèle statique c'est-à-dire à l'équilibre afin de définir l'énergie liée au matériau. L'étude des configurations d'équilibre pour une fonctionnelle donnée ou la recherche d'une fonctionnelle permettant d'obtenir des formes particulières est un domaine à part entière (voir par exemple [24], [45]). Nous donnons dans cette section une explication rapide des modèles de Korteweg utilisés pour les fluides compressibles dans la dynamique des plasmas. Le lecteur intéressé pourra consulter les travaux [48], [49]. Nous espérons par ce paragraphe sensibiliser le lecteur néophyte sur la nécessité dans certains problèmes d'avoir recours à une énergie supplémentaire et sur la difficulté d'en trouver une expression qui permet de décrire des solutions physiques attendues.

La première étude sur les 'interfaces liquide-vapeur revient à la théorie de Young-Laplace. On considère alors deux phases séparées par une hypersurface sans aucune dynamique, donc avec un saut de pression à l'interface (hypersurface) proportionnel à la courbure de l'hypersurface. C'est un problème à frontière libre. Une solution pour appréhender ce problème revient à rechercher la configuration d'équilibre comme un problème de minimisation de fonctionnelle d'énergie. Mais beaucoup de solutions existent sur ce type de problèmes dont certaines non physiques. Il est donc nécessaire de sélectionner une solution. Van der Waals semble avoir été le premier à avoir rajouté un terme lié à l'énergie de surface (interface diffuse) qui est thermodynamiquement consistant avec l'équilibre, voir par exemple [54], [29], [34].

$\mathrm{Au}$ niveau de la dynamique liquide-vapeur, on considère un ensemble d'équations de Navier-Stokes compressible avec loi d'état: pression dans chaque phase (loi d'état de Van der Waals). Mais les équations de Navier-Stokes ne sont pas compatibles avec les problèmes de phase multiples car il n'y a pas de terme de tension de surface. Korteweg a alors étendu Navier-Stokes en incluant une contribution dans le tenseur des contraintes qui tient compte de la tension de surface. On a alors un terme en plus lié à l'énergie de surface ajouté à la fonctionnelle d'énergie libre standard pour obtenir un équilibre correct. Le problème est le choix du terme supplémentaire à rajouter.

\section{1. Équilibre statique et minimisation d'énergie.}

Soit un fluide dans $\Omega \subset \mathbb{R}^{3}$ à température constante. On suppose le fluide sous deux formes : vapeur et liquide. On considère une densité du fluide notée $\rho$ dépendant de la variable spatiale $x$ dans $\Omega$ à valeurs dans ] $0, b[$. Pour décrire les deux phases, on note la fonction spécifique d'énergie libre $f$ de $] 0, b$ à valeurs dans $\mathbb{R}$. On définit la densité d'énergie suivante

$$
\pi(\rho)=\rho f(\rho) \text { avec } \rho \in] 0,+\infty[\text {. }
$$

On fait les hypothèses suivantes sur $\pi$ :

- i) $\left.\exists \alpha_{1}, \alpha_{2} \in\right] 0, \infty\left[: \alpha_{1}<\alpha_{2}\right.$ avec $\pi^{\prime \prime}>0$ dans $] 0, \alpha_{1}[\cup] \alpha_{2}, \infty\left[\right.$ et $\pi^{\prime \prime}<0$ dans $] \alpha_{1}, \alpha_{2}[$.

- ii) $\lim _{s \rightarrow 0} \pi(s)=\lim _{s \rightarrow+\infty} \pi(s)=\infty$

- iii) $\pi \geq 0$ dans $] 0,+\infty[$. 
Il existe une fonction affine unique $\ell=\ell(s)$ avec pente $\bar{c}:=\ell^{\prime}(s)$ et deux nombres $\left.\beta_{1} \in\right] 0, \alpha_{1}\left[, \beta_{2} \in\right] \alpha_{2},+\infty[$ tel que

$$
\ell\left(\beta_{i}\right)=\pi\left(\beta_{i}\right), \quad \pi^{\prime}\left(\beta_{i}\right)=\bar{c} .
$$

La fonction $\pi$ est convexe sur deux intervalles disjoints qui correspondent aux deux phases du fluide.

Phases et état de Maxwell : Soit $\rho: \Omega \rightarrow(0,+\infty)$ une distribution de densité.

- i) Si $\rho \in] 0, \alpha_{1}\left[,\left(\rho \in\left(\alpha_{1}, \alpha_{2}\right)\right)\right.$ ou $\left(\rho \in\left[\alpha_{2},+\infty\right)\right)$, on dit respectivement que le fluide en $x$ est en phase liquide, (en régime elliptique ou spinoidal) ou en phase vapeur.

- ii) Les nombres $\beta_{1}$ et $\beta_{2}$ sont appelés état de Maxwell.

Par la théorie thermodynamique, on définit la pression $p$ par

$$
p(\rho)=\rho^{2} \frac{\partial f}{\partial \rho}(\rho) \text { avec } \rho \in(0,+\infty) .
$$

Comme par définition $\pi(s)=s f(s)$ on a

$$
p(s)=s \pi^{\prime}(s)-\pi(s)
$$

donc

On obtient alors l'expression

$$
p^{\prime}(s)=\rho \pi^{\prime \prime}(s)
$$

$$
\pi(\rho)=\rho \int_{\bar{\rho}}^{\rho} \frac{p(s)}{s^{2}} d s
$$

avec $\bar{\rho}$ une densité constante de référence. La pression est une fonction ni convexe, ni monotone. On l'appelle pression de Van der Waals. Les branches convexes de $\pi$ correspondent aux endroits où $p$ est croissante. Notons que

$$
p\left(\beta_{1}\right)-p\left(\beta_{2}\right)=\left(\beta_{2}-\beta_{1}\right) \bar{c}-\pi\left(\beta_{2}\right)-\pi\left(\beta_{1}\right)=0 .
$$

Dans la pratique $p$ est mesurée et $f$ est trouvée ensuite.

Un exemple simple : $p(\rho)=\frac{R T_{*} b \rho}{b-\rho}-a \rho^{2}$ avec $R, T_{*}$ des constantes. Dans ce cas

$$
\pi(\rho)=R T_{*} \rho \ln \left(\frac{b \rho}{b-\rho}\right)-a \rho^{2}+c\left(T_{*}\right) .
$$

Problème variationnel : Soit une masse totale $m=\int_{\Omega} \rho(x) d x$ où $\Omega$ borné avec $|\Omega|=1$. Si $m$ est proche de 0 , on considère que l'on est en phase vapeur. Si $m$ est proche de 1 , on considère que l'on est en phase liquide. Pour $m$ intermédiaire, on peut parler de bulle vapeur dans liquide (bulle) et on peut parler de bulle liquide dans vapeur (goutte).

L'approche interface raide. On considère l'ensemble suivant

$$
A^{0}=\left\{\rho \in L^{1}(\Omega): \pi(\rho) \in L^{1}(\Omega), \int_{\Omega} \rho(x) d x=m\right\} .
$$

Le problème est alors de trouver l'équilibre statique, où l'on cherche $\rho^{0} \in A^{0}$ qui minimise la fonctionnelle d'énergie

$$
F^{0}=F^{0}(\rho)=\int_{\Omega} \pi(\rho) d x
$$

avec $\rho \in A^{0}$. Si on rajoute une fonction affine à $\pi(\rho)$, on ne change pas l'ensemble de minimiseur et donc en utilisant la fonction affine $\ell$, on peut supposer que

$$
\pi\left(\beta_{1}\right)=\pi\left(\beta_{2}\right)=0 .
$$


Remarque importante : Les minimiseurs ne sont donc pas uniques. Même en négligeant les minimiseurs obtenus par translation de minimiseurs. Toute fonction qui prend comme valeurs $\beta_{1}$ ou $\beta_{2}$ p.p. et obéit à $\int_{\Omega} \rho=m$ est minimiseur. Certains minimiseurs peuvent être relevant pour solide-solide mais pas pour un fluide. Il y a donc nécessité d'une sélection intelligente. Tout minimiseur de $F^{0}$ réalise une transition de phase comme un saut.

Modification de l'énergie. La solution est donc de modifier $F^{0}$ dans la région elliptique c'est-à-dire dans la région à deux phases. Nous présentons ici deux types de modifications possibles : une approche locale et une approche globale.

1) Approche locale. Soit l'ensemble

$$
A^{\text {local }}=H^{1}(\Omega) \cap A^{0} .
$$

Soit $\epsilon>0$ et $\gamma$ une constante de capillarité donnée. On regarde une fonction $\rho^{\epsilon}$ dans $A^{\text {local }}$ minimisant

$$
F_{\text {local }}^{\epsilon}=F_{\text {local }}^{\epsilon}(\rho)=\int_{\Omega} \pi(\rho)+\gamma \frac{\epsilon^{2}}{2}|\nabla \rho|^{2}
$$

avec $\rho \in A^{\text {local }}$. Des fonctions qui ont des sauts n'appartenant pas à $H^{1}(\Omega)$, on sait que $\rho^{\epsilon}$ change de phase si et seulement si on se trouve dans la région elliptique. Le lien mathématique entre interface diffuse et interface raide peut alors être étudié en regardant la limite quand $\epsilon$ tend vers 0 . Il s'agit des résultats de Modica-Mortola, voir [42] et [41]. On déduit facilement à $\epsilon$ fixé l'équation d'Euler-Lagrange associée.

Remarque importante : Une régularisation permet une sélection des solutions. Reste à savoir quelle genre de régularisation est physiquement raisonnable par rapport à l'application visée.

2) Approche non locale. Nous allons tout d'abord définir une fonction régularisante particulière.

Définition. Soit $d \in\{1,2,3\}$.

i) Une fonction radiale positive $\Phi \in L^{1}\left(\mathbb{R}^{d}\right)$ est appelée potentiel d'interaction dans $\mathbb{R}^{d}$ si $\Phi$ satisfait

$$
\int_{\mathbb{R}^{d}} \Phi(x) d x=1
$$

et

$$
\int_{\mathbb{R}^{d}} \Phi(x)|x| d x+\int_{\mathbb{R}^{d}} \Phi(x)|x|^{2} d x<\infty .
$$

ii) Pour un potentiel d'interaction $\Phi$ et $\epsilon>0$, on définit un potentiel d'interaction renormalisé $\Phi_{\epsilon}$ dans $\mathbb{R}^{d}$ par

$$
\Phi_{\epsilon}(x)=\frac{1}{\epsilon^{d}} \Phi\left(\frac{x}{\epsilon}\right) .
$$

On définit $A^{\text {global }}=A^{0} \cap L^{2}$ et $\rho^{\epsilon} \in A^{\text {global }}$ minimisant la fonctionnelle de Van der Waals

$$
F_{\text {global }}^{\epsilon}=F_{\text {global }}^{\epsilon}(\rho)=\int_{\Omega}\left(\pi(\rho)+\frac{\gamma}{4} \int_{\Omega} \Phi_{\epsilon}(x-y)(\rho(y)-\rho(x))^{2} d y\right) d x
$$

avec $\rho \in A^{\text {global }}$. Le terme non local pénalise la variation rapide si le support est dans la boule. Le $A^{\text {global }}$ contient des sauts malgré des valeurs dans la partie elliptique. La limite quand $\epsilon$ tend vers 0 peut être étudiée (cf. [1]) et l'équation d'Euler-Lagrange associée donnée.

Remarque importante. La fonctionnelle locale se retrouve à partir de la fonctionnelle non locale. Soit $F_{\text {global }}^{\epsilon}$ avec $\Omega=\mathbb{R}$. Supposons $\rho \in \mathcal{C}^{\infty}(\mathbb{R})$ une fonction analytique que l'on développera en série par rapport à $y$ au voisinage de $x$. On injecte l'expression dans $F_{\text {global }}^{\epsilon}$ et on fait le changement de variable $r:=(x-y) / \epsilon$.

$$
\int_{\mathbb{R}} \int_{\mathbb{R}} \Phi(r)\left(\sum_{k=1}^{\infty} \frac{1}{k !} \epsilon^{k} r^{k} \frac{\partial^{k}}{\partial x^{k}} \rho(x)\right)^{2} d x d r \approx \int_{\mathbb{R}} \int_{\mathbb{R}} \epsilon^{2} \Phi(r)\left(r \partial_{x} \rho(x)\right)^{2} d x d r .
$$


On s'intéresse alors à l'ordre principale en $k$. Remarquons que $\int_{\mathbb{R}^{3}} \Phi(r)|r|^{2} d r<+\infty$. Notons que Van der Waals a utilisé, dans son papier [54], $F_{\text {nonlocal }}$ et il a ensuite procédé à la simplification en supposant $\rho$ suffisamment régulier.

Remarque. L'exemple que nous venons de donner ici doit permettre au lecteur de comprendre que le choix de l'énergie libre n'est pas sans effet et qu'une analyse multi-échelles est souvent nécessaire (voir par exemple [35], $[5])$.

\subsection{Modèle dynamique.}

Dans cette partie, nous allons montrer que la prise en compte d'effet de capillarité au sein des équations des moments peut s'accompagner d'un choix bien particulier de viscosités si l'on désire obtenir des informations supplémentaires sur la densité. Nous considérerons pour cela le modèle de Korteweg compressible. Le lecteur intéressé est renvoyé à [3], [6], [13]. Nous expliquerons pourquoi une telle chose semble hors de portée pour le modèle de Korteweg incompressible.

\subsubsection{Modèles de Korteweg compressible.}

On suppose $\Omega$ comme étant l'espace entier ou le tore en dimension 1, 2 ou 3 . On considère le modèle suivant

$$
\begin{aligned}
& \partial_{t} \rho+\operatorname{div}(\rho u)=0 \\
& \begin{aligned}
\partial_{t}(\rho u)+\operatorname{div}(\rho u \otimes u)-2 \operatorname{div}(\mu(\rho) D(u)) \\
\quad-\nabla(\lambda(\rho) \operatorname{div} u)-\sigma \rho \nabla\left(\mu^{\prime}(\rho) \Delta(\mu(\rho))\right)+\nabla p(\rho)=0
\end{aligned}
\end{aligned}
$$

où $\sigma$ est supposé constant et $p$, la pression du fluide, est fonction de la densité. L'énergie assocée à ce système est donnée par

$$
\frac{1}{2} \frac{d}{d t} \int_{\Omega}\left(\rho|u|^{2}+\sigma|\nabla(\mu(\rho))|^{2}\right)+\frac{d}{d t} \int_{\Omega} \pi(\rho)+2 \int_{\Omega} \mu(\rho)|D(u)|^{2}+\int_{\Omega} \lambda(\rho)|\operatorname{div} u|^{2}=0
$$

avec $\pi(\rho)$ le potentiel de pression donné par

$$
\pi(\rho)=\rho \int_{\bar{\rho}}^{\rho}\left(p(s) / s^{2}\right) d s
$$

où $\bar{\rho}$ est une densité constante de référence. Il est montré dans [12], que si

$$
\lambda(\rho)=2\left(\mu^{\prime}(\rho) \rho-\mu(\rho)\right)
$$

alors on a également un nouveau type d'égalité d'énergie

$$
\begin{aligned}
\frac{1}{2} \frac{d}{d t} \int_{\Omega}(\rho \mid u+ & \left.\left.2 \nabla(\phi(\rho))\right|^{2}+\sigma|\nabla(\mu(\rho))|^{2}\right)+\frac{d}{d t} \int_{\Omega} \pi(\rho)+2 \int_{\Omega} \mu(\rho)|A(u)|^{2} \\
& +\int_{\Omega} p^{\prime}(\rho) \mu^{\prime}(\rho)\left[\left.\nabla \sqrt{\rho}\right|^{2}+\int_{\Omega} \sigma \mu^{\prime}(\rho)|\Delta(\mu(\rho))|^{2}=0\right.
\end{aligned}
$$

où $A(u)=\left(\nabla u-{ }^{t} \nabla u\right) / 2$ et $\rho \phi^{\prime}(\rho)=\mu^{\prime}(\rho)$. 
Notons que dans la première égalité, on retrouve l'énergie libre de surface de Korteweg $\int_{\Omega} \sigma|\nabla(\mu(\rho))|^{2}$. Le force capillaire associée à cette énergie est donnée en calculant la dérivée temporelle suivante

$$
\begin{aligned}
\frac{1}{2} \frac{d}{d t} \int_{\Omega}|\nabla(\mu(\rho))|^{2} & =\int_{\Omega} \nabla(\mu(\rho)) \cdot \nabla\left(\mu^{\prime}(\rho) \partial_{t} \rho\right) \\
& =-\int_{\Omega} \nabla(\mu(\rho)) \cdot \nabla\left(\mu^{\prime}(\rho) \operatorname{div}(\rho u)\right) \\
& =-\int_{\Omega} \rho u \cdot \nabla\left(\mu^{\prime}(\rho) \Delta \mu(\rho)\right) .
\end{aligned}
$$

On obtient alors la force donnée par $-\sigma \rho \nabla\left(\mu^{\prime}(\rho) \Delta \mu(\rho)\right)$. L'égalité formelle d'énergie utilise cette identité. On verra, par la suite, dans les applications biologiques une force capillaire élastique due à la déformation de la matrice extra-cellulaire, voir (4.16).

\subsubsection{Modèles de Korteweg incompressible.}

Une version incompressible du modèle de Korteweg s'écrit

$$
\begin{aligned}
& \partial_{t} \rho+\operatorname{div}(\rho u)=0, \\
& \begin{array}{l}
\partial_{t}(\rho u)+\operatorname{div}(\rho u \otimes u)-2 \operatorname{div}(\mu(\rho) D(u)) \\
\operatorname{div} u=0 .
\end{array}
\end{aligned}
$$

Montrer des résultats mathématiques probant comme l'existence globale de solutions faibles ou l'existence globale de solutions fortes pour données proches de l'équilibre est encore ouvert. La structure incompressible semble plus difficile que la structure compressible. Notons que l'on peut rencontrer des modèles semblables dans les problèmes de déplacements de vésicules dans un fluide où $\rho$ serait en fait la fonction décrivant l'interface, voir [21]. La difficulté réside dans la contrainte d'incompressibilité qui empêche certains choix de fonctions tests, notamment celles utilisés dans le cas compressible pour obtenir la nouvelle égalité d'énergie (3.11).

\section{Application À la modélisation de processus CancÉreux}

Nous allons exhiber dans cette section divers exemples d'applications de méthodes de paramètre d'ordre à la modélisation de processus cancéreux.

\subsection{Modèles multi-espèces.}

Pour décrire l'évolution d'une tumeur, il faut différencier différents types de cellules mises en jeu comme par exemple les cellules mortes, cancéreuses, quiescentes, saines. Chaque entité étant définie par une dynamique. Une théorie classique peut alors être utilisée, voir par exemple [15], [25], [47], [14] mais également une théorie de mélange, voir par exemple [2]. Cette théorie dépend fortement des moyennes considérées et des potentiels thermodynamique choisis. Le lecteur interessé est renvoyé à [51].

Méthode classique. Dans le cadre d'une application d'un modèle de la croissance cancéreuse, considérons donc par exemple $P$ la densité de cellules proliférantes, $Q$ la densité de cellules quiescentes, $D$ la densité de cellules mortes par nécrose. On a les équations suivantes

$$
\begin{aligned}
& \partial_{t} P+\operatorname{div}\left(v_{P} P\right)=\Gamma_{P}, \\
& \partial_{t} Q+\operatorname{div}\left(v_{Q} Q\right)=\Gamma_{Q}, \\
& \partial_{t} D+\operatorname{div}\left(v_{D} D\right)=\Gamma_{D},
\end{aligned}
$$


où $\Gamma_{j}$ désigne le rapport entre naissance et mort de chaque individu et $v_{j}$ la vitesse des cellules de type $j$. Les rapports donneront la dynamique entre les diverses populations. Ils dépendent, il va de soit, de concentration d'espèces chimiques comme les nutriments, l'oxygène, les chemo-attractants notés $c_{i}$ qui satisfont des équations de type advection-diffusion

$$
\partial_{t} c_{i}+\operatorname{div}\left(c_{i} v\right)=\operatorname{div}\left(Q_{i} \nabla c_{i}\right)+\left(G_{i}-D_{i}\right) c_{i}
$$

où $Q_{i}$ est le coefficient de diffusion, $v$ la vitesse d'advection, $G_{i}$ le terme de production et $D_{i}$ le terme de dégradation.

On peut complexifier les EDPs considérées en prenant en compte le parcours des cellules dans le cycle cellulaire avec une modélisation structurée en âge en sus. Cela a été fait par exemple dans [14] et [47].

La dernière étape consiste à décrire l'évolution tumorale c'est-à-dire la frontière libre qui en décrit le contour. On considère en fait la densité de cellules saines de la matrice extra-cellulaire, notée $M$ solution de

$$
\partial_{t} M+\operatorname{div}\left(v_{M} M\right)=0
$$

et on fait l'hypothèse que la densité totale de cellule est constante

$$
P+D+N+M=n_{0}=\text { Cste. }
$$

Cette contrainte va permettre de simplifier le système lorsque l'on considère que les cellules se déplacent avec une même vitesse $v$. On se retrouve alors avec une équation sur $n_{0} \operatorname{div} v=\Gamma_{P}+\Gamma_{Q}+\Gamma_{D}$. Une approche classique consiste maintenant à utiliser une loi de Darcy qui décrit les écoulements en milieux poreux. Ceci conduit à supposer que le champ de vitesse $v$ découle d'un potentiel $p$

$$
v=-K \nabla p
$$

On obtient alors une équation elliptique sur la pression.

Méthode de la théorie des mélanges. Dans ce cas, une dynamique est prescrite sur les vitesses des entités mises en jeu. On est alors en présence d'un couplage équation de masse, équation des moments comme dans la seconde partie. L'ensemble des cellules est supposé vivre dans un environnement liquide dans lequel il y a diffusion de certains facteurs chimiques. On peut supposer par exemple que l'amas tumoral est constitué d'un seul type de cellule comme cela a été fait dans [2]. On peut alors écrire un système du type

$$
\begin{aligned}
& \partial_{t} \phi_{T}+\operatorname{div}\left(\phi_{T} u_{T}\right)=\Gamma_{T}, \\
& \partial_{t} \phi_{\ell}+\operatorname{div}\left(\phi_{\ell} u_{\ell}\right)=\Gamma_{\ell}, \\
& \rho_{T} \phi_{T}\left(\partial_{t} u_{T}+u_{T} \cdot \nabla u_{T}\right)-\operatorname{div} \sigma_{T}=m_{T}, \\
& \rho_{\ell} \phi_{\ell}\left(\partial_{t} u_{\ell}+u_{\ell} \cdot \nabla u_{\ell}\right)-\operatorname{div} \sigma_{\ell}=m_{\ell},
\end{aligned}
$$

où $\Gamma_{T}, \Gamma_{\ell}$ désignent la production de cellules tumorales et de liquide, $\sigma_{\ell}$ et $\sigma_{T}$ sont les tenseurs de contraintes associés au liquide et à la tumeur. Les termes $m_{\ell}$ et $m_{T}$ contiennent par exemple des termes de frottement entre constituants. On s'inspire alors de la méthode expliquée dans la seconde partie pour se ramener à un système sur des quantités moyennes en exprimant (de manière plus ou moins ad-hoc) l'écart relatif des vitesses en fonction des vitesses moyennes. On parle alors d'un choix de fermeture à faire. Il va de soit qu'il serait très intéressant de comparer sur des cas tests l'effet de divers choix.

Remarquons également qu'il est possible de s'inspirer de [11] afin de pouvoir par exemple écrire un modèle de mélange en différenciant deux espèces de cellules dans l'amas tumoral par exemple les proliférantes et les quiescentes.

\subsection{Modèles de dynamique de cellules}

Si la composition de la cellule est désormais relativement bien connue, sa complexité en terme de rhéologie reste un défi [55]. On peut fondamentalement distinguer trois éléments : un noyau entouré du cytoplasme, lui 
même ceint d'une membrane dotée d'une perméabilité contrôlée. Le noyau est essentiellement visco-élastique, le cytoplasme peut être assimilé à un gel dont les constituants peuvent polymériser et dépolymériser (comme l'actine par exemple) selon les conditions, la membrane est élastique (surface faiblement déformable) et possède une énergie de courbure. Des récepteurs sur cette membrane peuvent créer des liaisons avec l'extérieur (par exemple, les cellules voisines ou bien la matrice extra-cellulaire MEC), de même les protéines du cytoplasme peuvent s'insérer dans la membrane et former de telles liaisons, ce qui modifie les propriétés de celle-ci [16]. Modéliser la migration d'une cellule dans un vaisseau sanguin en tenant compte de termes de liaison pour simuler le contact cellule/cellule, l'étalement puis l'extravasation (c.a.d la transmigration cellulaire) est primordial. Une prise en compte de la cinétique de formation-rupture des liaisons [22], [33], [28] est indispensable si l'on désire mieux appréhender les phénomènes de formation de métastases. Actuellement, des modèles simples à double couches pour modéliser la cellule existent. Les méthodes de lignes de niveaux avec terme de tension de surface et force de traction sont également utilisés pour appréhender les phénomènes adhésifs. On rencontre par exemple dans la littérature le modèle viscoélastique suivant [31] :

$$
\begin{aligned}
& \partial_{t} \phi+u \cdot \nabla \phi=0, \\
& \operatorname{div} u=0, \\
& \rho\left(\partial_{t} u+u \cdot \nabla u\right)-2 \operatorname{div}(\eta(\rho) D(u))-\operatorname{div} T+\nabla p \\
& \quad-\alpha \delta(\phi) \kappa n-F_{b} \delta(\phi)=\rho g,
\end{aligned}
$$

avec des conditions aux bords de type non-glissement sur la paroi vasculaire (composée de cellules endothéliales) modélisée par un mur pour simplifier et sur le bord de la cellule. Pour plus de renseignements sur les fluides viscoélastiques, voir [32] et pour la mise en place des méthodes de lignes de niveaux pour des fluides complexes, [46]. On note $\delta(\phi)$ la mesure de lebesgue sur la sous variété $\phi^{-1}\{0\}$. Le tenseur des contraintes $T$ est donné par $T=c \mu_{s} A / \lambda_{r}$ où $\lambda_{r}$ est le temps de relaxation, $\eta_{s}$ la viscosité du solvent, $c$ la concentration du polymère. Le cytoplasme est ainsi assimilé à une solution de polymères dans un solvant. Le tenseur de configuration $A$ est donné par la loi d'Oldroyd

$$
\partial_{t} A+u \cdot \nabla A=A(\nabla u)^{t}+\nabla u A-\frac{1}{\lambda_{r}}(A-\mathrm{Id}) .
$$

Notons dans l'équation de quantité de mouvement le terme maintenant classique de tension de surface [46] vu dans la première partie et le terme nouveau $F_{b}$ qui va permettre de modéliser l'attraction ou la répulsion entre cellule/cellules endothéliales (le bord du domaine). Une possibilité est d'utiliser un terme $F_{b}$ dérivant d'un potentiel. Plus précisemment, on définit un potentiel d'adhésion entre le mur et la cellule de la forme [52] :

$$
W(x)=w\left(d_{0} / x\right)^{2}\left(\left(d_{0} / x\right)^{2}-2\right)
$$

où $d_{0}$ est la longueur d'adhésion, $x$ la distance au bord. Pour $x \gg d_{0}$ le potentiel est attractif, et si $x \ll d_{0}$ le potentiel devient répulsif. On écrit alors $F_{b}=-d W / d x$. On peut également définir une dynamique de liaison en utilisant un modèle cinétique et considérer une énergie de liaison élastique liée à cette inconnue, voir les travaux de Dembo et al. [22] ou Khismatullin et al. [33]. On définit alors un force $F_{b}$ proportionnelle au nombre de liaisons de type $N$ et on écrit une Équation Différentielle Ordinaire (EDO) pour $N$ qui permet de représenter la création et la disparition de liaisons. Une autre approche a été proposé par Jadhav et al. [28] qui utilisent une cellule dotée d'une membrane élastique (non linéaire) entourant un cytoplasme Newtonien. Dans ce travail, ils proposent un modèle stochastique de formation-dissociation de liaisons entre les récepteurs et les ligands présents respectivement sur la paroi et sur le leucocyte. Ils retrouvent par exemple des phénomènes de roulement et d'étalement de cellules soumises à un cisaillement dans un canal. Ces phénomènes sont actuellement en cours d'étude par les auteurs [31]. Notons que la dimension d'une cellule est parfois comparable au diamètre du vaisseau, nous entrons alors dans le cadre de la microfluidique. Les travaux récents [26] devrait permettre de contrôler les oscillations possibles en présence de fortes tensions de surface. 




(a)

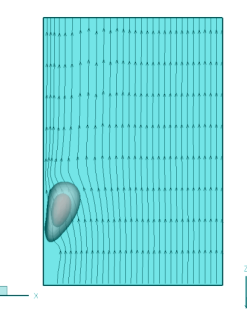

(b)

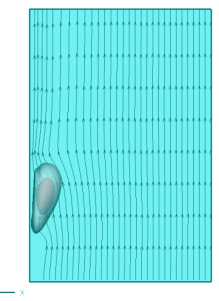

(c)

Fig 1 : Adhésion et déformation d'un leukocyte

Nous présentons ici une illustration du modèle décrit précédemment sur l'adhésion et la déformation d'une cellule. Les trois images sont respectivement prise $t=0.82, t=2.46$ et $t=3.94$. Par simplicité nous ne donnerons pas ici les paramètres et nous renvoyons le lecteur à [31] pour plus de détails.

\subsection{Modèle d'invasion}

Dans [14], nous présentons un modèle mathématique de croissance tumorale avasculaire et son étude numérique en 2 et 3 dimensions d'espace. Pour cela, on utilise un modèle multi-échelles d'équations aux dérivées partielles pour décrire l'évolution des densités de cellules cancéreuses. Ces équations prennent en compte le cycle cellulaire contrôlé par des facteurs environnementaux comme l'hypoxie et la surpopulation. La croissance tumorale crée un mouvement avec une vitesse correspondante. Pour fermer ce système, on utilise l'équation de Stokes pour cette vitesse. La matrice extra-cellulaire (ECM), avec laquelle les cellules bougent et se dupliquent interagit mécaniquement avec la tumeur. On utilise alors un méthode de lignes de niveaux pour décrire la membrane en utilisant une énergie de surface inspirée de [19]. La méthode de lignes de niveaux pour la modélisation de la croissance tumorale a été utilisée dans [37], [56], [39] et [38]. L'originalité dans [14] est de considérer une dégradation possible de la membrane et un champ de vitesse qui n'est pas incompressible. Une application de ce type de modèle mathématique à l'étude du bénéfice thérapeutique d'agents anti-invasifs a été réalisé dans [47]. L'évolution de l'oxygène (pour estimer l'hypoxie) est décrite par une équation de diffusion.

L'énergie de surface de la membrane est supposée donnée par

$$
\mathcal{E}(X)=\int_{\Gamma_{t}} \eta^{+}(t, x) E(J(t, x)|\nabla \phi(t, x)|) J^{-1}(t, x) d x
$$

avec $E(s)=T_{0}(s-1)$ où $s$ désignera le facteur d'étirement de la surface, $\eta$ une fonction permettant de décrire l'état de la membrane et où l'on note par $J$ le jacobien solution de

$$
\partial_{t} J+u \cdot \nabla J=\operatorname{div} u J
$$

Comme nous l'avons expliqué section 2, nous allons utiliser un artefact d'étalement de frontière pour se ramener à une énergie de volume. Plus précisement, nous utilisons l'énergie régularisée $\mathcal{E}_{\epsilon}$, dépendant de $\phi$ et $\eta$, suivante

$$
\mathcal{E}_{\epsilon}(t)=\int_{\Omega}|\nabla \phi| E(|\nabla \phi(t, x)| J(t, x)) \frac{1}{\epsilon} \zeta\left(\frac{\phi(t, x)}{\epsilon}\right) \eta^{+}(t, x) J^{-1}(t, x) d x
$$



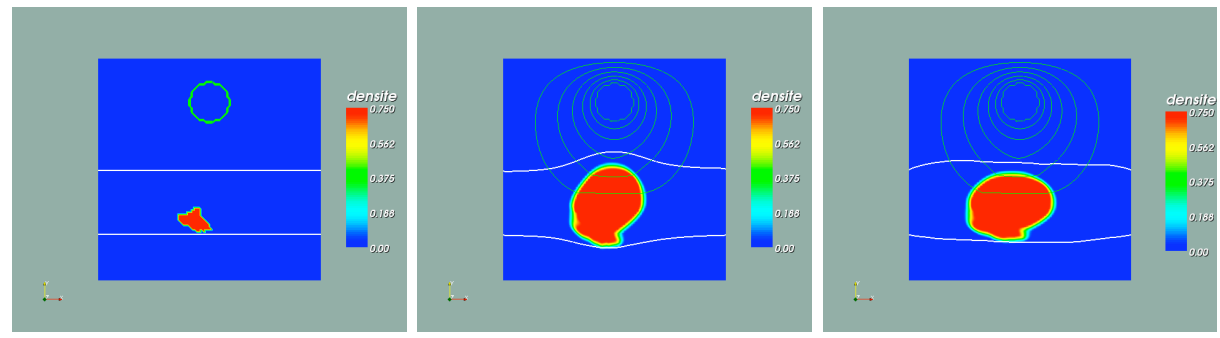

FiguRE 1. Déformation de membrane extra-cellulaire par croissance tumorale.

Nous présentons ici une illustration du modèle décrit rapidement dans la section précédente. Nous ne considérons pas ici de dégradation de membrane. Le graphique de gauche est la donnée initiale avec en rouge la tumeur, en trait jaune la membrane extra-cellulaire et le cercle supérieur correspond à la source extérieure d'oxygène. Le graphique centrale considère un déplacement de membrane sans tension de surface. Le dernier calcul prends en compte la tension de surface. Par simplicité nous ne donnerons pas ici les paramètres et nous renvoyons le lecteur à [14] pour plus de détails.

avec le terme $|\nabla \phi| \zeta(\phi(t, x) / \epsilon) / \epsilon$ qui est l'approximation de la mesure de Dirac sur $\Gamma_{t}$. En pratique, on prend $\epsilon=2 \delta$ où $\delta$ est le pas d'espace typique. La fonction régularisant $\zeta$ est définie, voir section 2, par

$$
\begin{aligned}
& \zeta(y)=(1+\cos \pi y) / 2 \text { si }|y|<1, \\
& \zeta(y)=0 \text { sinon. }
\end{aligned}
$$

On retrouve alors la contribution de cette énergie (régularisée) au niveau des Équations aux Dérivées Partielles en dérivant par rapport au temps. On écrit alors

$$
\frac{d}{d t} \mathcal{E}_{\epsilon}(t)=-\int_{\Omega} F(t, x) \cdot v(t, x) d x .
$$

La force $F$ donnera la force élastique à rajouter dans l'équation qui gouverne la dynamique de déplacement c'est-à-dire la vitesse $u$ satisfaisant

$$
-\operatorname{div}(\mu(\phi) D(u))+\nabla p=F .
$$

Dans notre cas, on trouve

$$
\begin{aligned}
F(t, x)=-T_{0} \eta^{+} \kappa & \frac{1}{\epsilon} \zeta\left(\frac{\phi}{\epsilon}\right) \nabla \phi \\
& +\left(E(J|\nabla \phi|)+T_{0}\right) \frac{1}{\epsilon} \zeta\left(\frac{\phi}{\epsilon}\right) J^{-1} \nabla \eta^{+} .
\end{aligned}
$$

La difficulté est alors de conserver les propriétés des variables $\phi$ et $\eta$ qui sont de valoir 0 ou 1.

Remerciements. Ce document fait suite à un mini-cours, donné par le premier auteur, dans le cadre du groupe de travail d'Orsay organisé par Stéphane LABBÉ en 2005 : "Quelques modèles multiphasiques : propriétés et difficultés liées à la capillarité et à la viscosité". Les auteurs tiennent à remercier Stéphane LABBÉ pour leur avoir proposé de rédiger cet article pour ce numéro spécial d'ESAIM Proc. et pour l'organisation des séances du groupe de travail numérique d'Orsay depuis plusieurs années. Nous remercions également le/la referee du papier qui par son travail exemplaire a permis d'améliorer très largement la précision et la présentation du document. 


\section{REFERENCES}

[1] G. Alberti, G. Belletini. A non local anisotropic model for phase transitions: asymptotic behavior of rescaled energy. European Journal of Applied Mathematics, 9, 261-284, (1998).

[2] D. Ambrosi, L. Preziosi. On the closure of mass balance models for tumor growth. Math. Models and Methods in Applied Sciences. 12, no. 5, 737-754, (2002).

[3] D.M. Anderson, G.B. MacFadden, A.A. Wheeler. Diffuse-interface methods in fluid mechanics. Ann. Rev. Fluid Mech. 30, 139-165, (1998).

[4] J. Beaucourt, T. Biben, A. Leyrat, C. Verdier. Modeling breakup and relaxation of newtonian droplets using the advected phase field approach. Soumis (2006).

[5] A. Belletini, C. Presetti. Surface tension in Ising systems with Kac potentials. J. Stat. Phys., 82, no 3/4, 743-793, (1996).

[6] S. Benzoni, R. Danchin, S. Descombes, D. Jamet. Structure of Korteweg models and stability of diffuse interfaces. Interfaces and Free Boundaries. Modelling, Analysis and Computation, 7, no. 4, 371-414, (2005).

[7] T. Biben, C. Misbah. Tumbling of vesicles under shear flow within an advected-field approach. Physical Review E, 67, (2003).

[8] T. Biben, C. Misbah, A. Leyrat, C. Verdier. An Advected-field approach to the dynamics of fluid interfaces. Europhysics letters. 63, (4), 623-629, (2003).

[9] F. Boyer. Non-homogeneous Cahn Hilliard fluids. Ann. Inst. H. poincaré Anal. Non linéaire. 18, no.2, 225-259, (2001).

[10] F. Boyer. Mathematical study of multi-phase flow under shear through order parameters formulation. Asymptot. Anal., 20, no.2, 175-212, (1999).

[11] F. Boyer, C. Lapuerta. Study of a three component Cahn-Hilliard flow model. À paraître dans M2AN, (2007).

[12] D. Bresch, B. Desjardins. Some diffusive capillary models of Korteweg type, C. R. Acad. Sciences, Paris, Section Mécanique. 332, 881-886, (2004).

[13] D. Bresch, B. Desjardins, C.K. Lin. On some compressible fluid models: Korteweg, lubrication and shallow water systems. Comm. Partial Differential Equations. 28, no.3-4, 843-868, (2003).

[14] D. Bresch, T. Colin, E. Grenier, B. Ribba, O. Saut. Computational modeling of solid tumor growth: the avascular stage. Soumis (2007).

[15] H. Byrne, L. Preziosi. Modelling solid tumour growth using the theory of mixtures. Math. Medecine and Biology. 20, 341-366, (2003).

[16] E. Canetta, A. Leyrat, C. Verdier, A. Duperray. Measuring cell viscoelastic properties using a force spectrometer: influence of the protein-cytoplasm interactions. Biorheology, 42, 321-333, (2005).

[17] M.A.J. Chaplain, L. Graziano, L. Preziosi. Mathematical modelling of the loss of tissue compression responsiveness and its role in solid tumour development. Submitted (2005).

[18] L. Chupin. Mélange de fluides viscoélastiques. Thèse de doctorat, Bordeaux, (2003).

[19] G.-H. Cottet, E. Maitre. A level-set formulation of immersed boundary methods for fluid-structure interaction problems. C.R. Acad. Sci. Paris, section Mathématiques, 338(7) : 581-586, (2004).

[20] G.-H. Cоттет, E. Maitre. A level-set method for fluid-structure interactions with immersed surface. M3AS, à paraitre $(2007)$.

[21] G.-H. Cottet, E. Maitre, Th. Milcent. Eulerian formulation and level sets models for incompressible fluid structure interaction. En préparation (2007).

[22] M. Dembo, D.C. Torney, K. Saxman, D. Hammer. The reaction-limited kinetics of membrane-to-surface adhesion and detachment Proc. Roy. Soc. London - B. Biol. Sciences, 234, 55-83, (1988).

[23] J. Etienne, E. Hopfinger, P. Saramito. Numerical simulation of high density ratio lock-exchange flows, Physics of Fluids, $17(3),(2005)$.

[24] L.C. Evans, W. Soner, P.E. Souganidis. Phase transitions and generalized motion by mean curvature. Comm. Pure Appl. Math., 45, 1097-1123, (1992).

[25] A. Friedman. A hierarchy of cancer models and their mathematical challenges. DCDS, vol.4, Series B, 147-160, (2004).

[26] C. Galusinski, P. Vigneaux. Level-Set method and stability condition for curvature-driven flows. Soumis (2007).

[27] R. Gatignol, P. Seppecher. Modelisation of fluid-fluid interfaces with material properties. J. Mécanique Théorique et Appliquée. Numéro spécial, 225-247, (1986).

[28] S. Jadhav, C.D. Eggleton, K. Konstantopoulos. A 3-D Computational Model Predicts that Cell Deformation Affects Selectin-Mediated Leukocyte Rolling. Biophysical J., 88, 96-104, (2005).

[29] D. Jamet. Diffusive interface model in fluid mechanics. Ecole thématique. Modèles de champ de phase pour l'évolution de structures complexes. Les Houches, 27-31 Mars 2006.

[30] G.S. Jiang, D. Peng. Weighted ENO Schemes for Hamilton-Jacobi equations. SIAM J. Sci. Comput., vol. 21:6, 2126-2143, (2006).

[31] Q. Jin, C. Verdier, P. Singh, N. Aubry, R. Chotard-Ghodsnia, A. Duperray. Direct simulation of the migration of leukocytes in pressure driven flows. Soumis, J. Fluids Eng. (2006).

[32] D.D. Joseph. Fluid dynamics of viscoelastic liquids. Springer Verlag, (1990). 
[33] D.B. Khismatullin, G.A. Truskey. A 3D numerical study of the effect of channel height on leukocyte deformation and adhesion in parallel-plate flow chambers. Microvasc. Res., 68, 188-202, (2004).

[34] D.J. Korteweg. Sur la forme que prennent les équations du mouvement des fluides si l'on tient compte des forces capillaires causées par des variations de densité considérables mais continues et sur la théorie de la capillarité dans l'hypothèse d'une variation continue de la densité. Archives Néerlandaises de Sciences Exactes et Naturelles, 1-24, (1901).

[35] C. Le Bris. Systèmes multi-échelles, modélisation et simulation. Mathématiques \& Applications, Springer, SMAI (2005).

[36] C.K. Liu, J. Shen. A phase field model for the mixture of two incompressible fluids and its approximation by a Fourier-spectral method. Physica D. (2001).

[37] P.Tн. MAcklin. Numerical simulation of tumor growth and chemotherapy. Thèse de Doctorat, Université du Minnesota, (2003).

[38] P.Th. Macklin, J.S. Lowengrub. Evolving interfaces via gradients of geometry-dependent interior problems: applications to tumor growth. J. Comput. Phys., 203, (1), 191-220, (2005).

[39] P.Th. Macklin, J.S. Lowengrub. An improved geometric-aware curvature discretization for level set methods: Application to tumor growth. J. Comput. Phys., 125, 392-401, (2006).

[40] E. Maitre. Review of numerical methods for free interfaces. Ecole thématique. Modèles de champ de phase pour l'évolution de structures complexes. Les Houches, 27-31 Mars 2006.

[41] L. Modica. The gradient theory of phase transitions and the minimal interface criterion. Arch. Rational Mech. Analysis. 98, 123-142, (1987)

[42] L. Modica, M. Mortola. Un exemplo di Gamma-convergenza Boll. un. mat. Ital. B. (5), 14:1, 285-299, (1977).

[43] S. Osher, R. Fedkiw. Level set methods and dynamic implicit interfaces. Applied Matematical Sciences, vol. 153, Springer, (2003).

[44] S. Osher, J. Sethian. Fronts propagation with curvature-dependent speed: algorithmas based on Hamilton-Jacobi formulations. J. Comput. Phys., 79, 12-49, (1988).

[45] F. Otto, P. Penzler, A. Rätz, T. Rump, A. Voigt. A diffusive interface approximation for step flow in epithelial growth. Nonlinearity. 17:3, 477-491, (2004).

[46] S.B. Pillapakkam, P. Singh. A level set method for computing solutions to viscoelastic two-phase flow. J. Comp. Physics, 174, 552-578, (2001).

[47] B. Ribba, O. Saut, T. Colin, D. Bresch, E. Grenier, J.-P. Boissel. A multiscale mathematical model of avascular tumor growth to investigate the therapeutic benefit of anti-invasive agents. Journal of Theoretical Biology, 243, 532-541, (2006).

[48] C. RohDE. On local and non-local Navier-Stokes-Korteweg systems for liquid-vapour phase transitions. Zamm Z. Angew. Math. Mech., 85, 839-857, (2005).

[49] C. Rohde. Phase transitions and sharp-interface limits of the 1D-elasticity system with non-local energy. Interfaces free Bound., 7:1, 107-129, (2005).

[50] M. Sussman, P. Smereka, S. Osher. A level set approach for computing solutions to incompressible two-phase flow, $J$. Comput. Phys., 114, 146-159, (1994).

[51] K.R. Rajagopal, L. TaO. Mechanics of mixtures. Singapore: World Scientific, (1995).

[52] S. Sukumaran, U. Seifert. A Influence of shear flow on vesicles near a wall. Phys. Rev. E, 64 (2001).

[53] A.K. Tornberg. Interface tracking with application to multiphase flows. PhD thesis, Royal Institute of Technology, Stockholm, (2000).

[54] Van Der WaAls. Thermodynamische theorie des Kapillarität under Vorausstzung stetiger Dichtanderung. Z. Phys. Chem, $657-725,(1894)$.

[55] C. Verdier. Review: Rheological properties of living materials: from cells to tissues. J. Theor. Medicine, 5:(2), 67-91, (2003).

[56] X. Zheng, S.M. Wise, V. CRistini. Nonlinear simulation of tumor necrosis, neo-vascuarization and tissue invasion via an adaptive finite-element/level-set method. Bulletin of Mathematical Biology, 67, 211-259, (2005). 